\title{
Consumo de agua y «Huella Hídrica» de las ciudades españolas
}

\section{Water consumption and «Water Footprint» of Spanish cities}

\section{La consommation d'eau et "empreinte eau» des villes espagnoles*}

\author{
José Antonio Sotelo Navalpotro ${ }^{1}$ y Maria Sotelo Pérez
}

\begin{abstract}
RESUMEN
En las últimas décadas, España ha vivido un notable crecimiento urbano marcado por la dispersión por el territorio de zonas residenciales, de actividades económicas secundarias y terciaras, por el flujo de personas y mercancías, ... que demandan y disponen nuevos usos de los recursos hídricos, repercutiendo, de manera directa e indirecta en la aparición de nuevos modelos de «creación de ciudad». De este modo, en el presente estudio se realiza una estimación del consumo y las demandas de agua y de «Huella Hídrica», tanto a nivel económico como ambiental, y su vinculación con el desarrollo endógeno, describiendo los recursos hídricos utilizados, necesarios para la producción y consumo de bienes y servicios así como de las demandas de agua directa, en las ciudades españolas, en general, y, de las grandes urbes, en particular.
\end{abstract}

PAlabras Clave: huella hídrica; agua virtual; consumo de agua; jerarquía urbana; desarrollo endógeno.

* La presente investigación se enmarca en el Proyecto de Investigación MINECO (2014). CTM2013-41750-P.

1 Instituto Universitario de Ciencias Ambientales (IUCA/UCM). Grupo de Investigación «Desarrollo y Gestión Ambiental del Territorio», Universidad Complutense de Madrid (UCM930539) (jasotelo@ucm.es) ORCID iD: http://orcid.org/0000-0003-2800-6677

2 Universidad Rey Juan Carlos (URJC). Grupo de Investigación «Desarrollo y Gestión Ambiental del Territorio». ORCID iD: http://orcid.org/0000-0002-5541-7941

Copyright: (0) 2018 CSIC. Este es un artículo de acceso abierto distribuido bajo los términos de la licencia de uso y distribución Creative Commons Reconocimiento 4.0 Internacional (CC BY 4.0). 
Abstract

In the last decades, Spain has experienced a remarkable urban growth marked by dispersion through the territory of residential areas, of secondary and tertiary economic activities, by the flow of people and goods,..., that demand and offer new uses of water resources, an impact, directly and indirectly in the emergence of new models of «city building». In this way, in the present study an estimate of consumption and water demands and "water footprint» is performed, both an economic and environmental level, and its relationship with endogenous development, describing water resources used, necessary for the production and consumption of goods and services as well as direct water demands in Spanish cities, in general, and, in big cities, in particular.

KEY WORDS: water footprint; virtual water; water consumption; urban hierarchy; endogenous development.

Cómo citar este artículo/Citation: Sotelo Navalpotro, José Antonio, Sotelo Pérez, Maria (2018): "Consumo de agua y «Huella Hídrica» de las ciudades españolas", Estudios Geográficos, LXXIX/284, pp. 115-140

\section{INTRODUCCIÓN}

Hace casi cuarenta años Kevin Lynch escribía: «imaginémonos por un instante que el crecimiento de la población y la evolución de la tecnología han conducido a la urbanización de todo el globo terráqueo», y se preguntaba, «¿cómo podríamos hacer de ese mundo totalmente «urbano» un lugar más humanizado?» (Lynch, K, 1965., Cities., Alfred A. Knopf, Inc.). De este modo, nos aproximaba a la idea de que el desarrollo de los territorios y, más concretamente, de las ciudades depende de la disponibilidad de recursos humanos, naturales y económicos, y, sobre todo, de los efectos de la interacción entre las fuerzas y mecanismos del desarrollo endógeno, en general, y, entre los efectos económicos que producen la organización flexible de la producción, la difusión de las innovaciones, el desarrollo urbano del territorio, y la dinámica institucional, en particular, desempeñando un papel fundamental el agua.

En este contexto, desde el punto de vista de la producción de espacios urbanos, se torna relevante el estudio de indicadores de sostenibilidad como la «Huella Hídrica», puesto que en las conurbaciones, en general, y en las españolas, en particular, la separación del espacio con funciones diferentes y la segregación social con densidades elevadas de personas con rentas, instrucción, etc., similares, obliga a un aumento del metabolismo externo. Se enmarca en el ámbito de las cinco salvaguardias que señala el profesor Carlos Jerez Mir (2011), «la pública, la social, la productiva, la ambiental y la programática», y 
representan los factores esenciales de la vida y del trabajo humano que deben ser defendidos y exaltados en la planificación del territorio; de forma concreta es en la salvaguardia ambiental de los recursos donde se debe poner especial énfasis en la preservación de los bienes naturales para el disfrute de todos los ciudadanos.

No debemos olvidar que los grandes problemas ambientales globales o mundiales tienen una raíz local, y eminentemente urbana, y su «huella» no tiene una única localización geográfica, pues la impronta puede afectar a sus propios espacios, a sus entornos geográficos más o menos inmediatos, $y$, a regiones remotas. Otras veces, no son tanto las ciudades como las estructuras territoriales urbanas, especialmente las ciudades «desparramadas», los crecimientos suburbanos, al parecer imparable, a los que se les señala como modelos de gran insostenibilidad. Lo cierto es que en muchos países, el crecimiento del espacio consumido por la expansión urbana, llega a ser bastante mayor al de la suma de todos los crecimientos, a lo largo de toda la historia urbana precedente. Unas veces acorde con su crecimiento demográfico, pero otras, en franca contradicción, como fue el caso de nuestro país, en las décadas anteriores a la crisis del 2008, y al crecimiento demográfico por inmigración (Sotelo Navalpotro, J.A. et alii, 2011). Con todo ello, es importante establecer la relación existente entre las grandes ciudades españolas y la «Huella Hídrica», asumiendo y superando las complejidades de cálculo y uso de los mismos, favoreciendo su aplicación por parte de instituciones y, posteriormente, por la propia sociedad.

\section{Objetivos}

El presente estudio tiene por objeto realizar una estimación de la «Huella Hídrica» de las ciudades española, en general, y de las grandes urbes, en particular, tanto a nivel económico como medioambiental, analizando de manera detallada una proyección territorial sobre las repercusiones que ésta demanda de recursos hídrico tiene en dichos territorios. De esta forma:

En primer lugar, se analiza y calcula la composición y el perfil de consumo de agua distribuida en España, por grandes sectores y conjuntos regionales existentes en las ciudades españolas.

En segundo lugar, se estudia la jerarquización urbana a través de su «Huella Hídrica» así como la correlación existente entre el agua y la ciudad, con el fin de conocer si las ciudades son los territorios que consumen mayores cantidades de recursos hídricos. 
En tercer, y último lugar, se examina el papel que desempeñan las ciudades en el conjunto del consumo hídrico, una vez incorporado el «Agua Virtual»; y la relación existente entre aquel y el agua de consumo doméstico.

\section{Fuentes y METOdOLOGÍA}

Estudiar la «Huella Hídrica» a niveles territoriales inferiores y específicos permite conocer exactamente cuánta agua, y en qué condiciones, se utiliza en los sistemas de agua locales, y cuánta agua sería necesaria para contrarrestar las corrientes contaminadas (Chapagain y Orr, 2009). El estudio emplea una metodología de cálculo de la «Huella Hídrica» desarrollada por Chapagain y Hoekstra (2004), actualizada en Hoekstra et alii. (2009), posteriormente, en Hoekstra et alii. (2011) y, por último, Sotelo Navalpotro, J.A. (2010, 2011, 2012) en los que se han establecido los estándares de cálculo a nivel mundial, y, español (autonómico, provincial y municipal). Nuestra metodología ha sido adaptada a los datos disponibles para realizar un análisis más detallado y preciso de los aspectos generales de la demanda de recursos hídricos en las ciudades españolas. Entre las principales fuentes estadísticas para realizar los cálculos del estudio, se han empleado las bases de datos del Institutos Nacionales de Estadística, Eurostat, el Ministerio de Agricultura, Alimentación y Medio Ambiente, del Banco Mundial, Aquastat-FAO y del «Water Footprint Network».

Se parte de la idea según la cual la «Huella Hídrica» total de un consumidor es la suma de su «Huella Hídrica» directa e indirecta, siendo la «Huella Hídrica» directa de un consumidor o productor, o de un grupo de consumidores o productores, el consumo de agua dulce y la contaminación asociada a su uso por el consumidor o el productor («Water Footprint Network», 2010).

Así, la «Huella Hídrica» de un consumidor (WFcons) es el volumen total de agua dulce consumida y contaminada, necesaria para la producción de los bienes y servicios consumidos por el consumidor. La «Huella Hídrica» de un grupo de consumidores es igual a la suma de la «Huella Hídrica» de los consumidores individuales. Se calcula sumando la «Huella Hídrica» directa de la persona y su «Huella Hídrica» indirecta:

$$
\text { WFcons }=\text { WFcons.dir }- \text { WFcons.indir }
$$

Donde:

- WFcons.dir es la «Huella Hídrica» directa, que se refiere al consumo y la contaminación del agua relacionada con su uso en el hogar o en el jardín. 
-WFcons.indir es la «Huella Hídrica» indirecta, que se refiere al consumo y la contaminación del agua asociada con la producción de los bienes y servicios consumidos por el consumidor. Es decir, el agua que se utilizó para producir la comida, la ropa, el papel, la energía y los bienes industriales consumidos.

El uso indirecto del agua se calcula multiplicando todos los productos consumidos por sus respectivas huellas hídricas:

$$
\text { WFcons.indir }=\Sigma p\left(C(p){ }^{*} W^{*} \text { prod }(p)\right)
$$

Donde:

- $C(p)$ es el consumo del producto $p$ (unidades del producto / tiempo).

- WFprod(p) es la «Huella Hídrica» de ese producto (volumen de agua / unidad de producto).

El volumen total consumido de $p$ generalmente procede de diferentes lugares $\mathrm{x}$.

La «Huella Hídrica» promedio de un producto consumido p es:

$$
W F_{p r o d}^{*}(p)=\frac{\sum_{x}\left(C(x, p) * W F_{p r o d}^{*}(x, p)\right)}{\sum_{x} C(x, p)}
$$

Donde:

- $C(x, p)$ es el consumo de productos $p$ procedentes de $x$ (unidades de producto/tiempo).

- WFprod(x,p) es la «Huella Hídrica» de los productos $p$ procedentes de $x$ (volumen de agua/unidad de producto).

De forma concreta, el cálculo de la «Huella Hídrica» de nuestro país, desde la realidad de la demanda, nos aproxima a la demanda de agua en los usos agrícolas, en los industriales y en los usos domésticos, el turismo y los servicios, desde la perspectiva de las grandes ciudades españolas.

Primeros ReSUltados. LAS CIUDADES ESPAÑOLAS Y SUS CONSUMOS DE AGUA

En un mundo en el que proliferan los indicadores de sostenibilidad, y, más concretamente, los relacionados con el agua como son la «Huella Hídrica» o el 
«Agua Virtual» se torna imprescindible la unificación y normalización tanto de cálculo, generación de bases estadísticas vs. cartográficas y aplicación, evitando y eliminando la dispersión de las mismas, cuestión ésta de notable importancia en nuestra investigación. Hoy por hoy, compleja a la par que imprescindible en cuanto al indicador «Huella Hídrica», puesto que aún no disponemos de los sistemas armonizados de indicadores de agua, con datos fiables para el uso, tanto de consumo de agua como de su precio, tal y como se establecían en los informes de «Planeta Vivo», que permita una óptima gestión y gobernanza de los recursos hídricos en nuestro país.

En dicho informe se establecía una «Huella Hídrica» de producción anual en España de $94 \mathrm{Km}^{3}$, y una «Huella Hídrica» «per cápita» de $2.325 \mathrm{~m}^{3} / \mathrm{hab} /$ año, donde el origen del «Agua Virtual» es un 64\% interna y un 36\% externa. En la presente investigación estudiamos el peso que tienen la mayor parte de las ciudades españolas en términos de comercio de «Agua Virtual», y su «Huella Hídrica».

El agua consumida en las ciudades es solo una parte de la «Huella Hídrica» que generan las mismas, y en principio, la que parece tener una mayor fiabilidad de las fuentes, sin que sea del todo exacto, ya que no todo el agua consumida es facturada y medida. La diferencia entre el agua distribuida por las redes públicas de abastecimiento, y los recursos hídricos «empleados de manera eficaz», complican mucho esta aproximación a la "Huella Hídrica» urbana - de hecho, se tiende a pensar que los mayores niveles de demanda de agua en las ciudades se corresponden con los usos industriales y domésticas, en vez de los servicios, pero ese es un problema de contabilidad ${ }^{4}$.

A partir de los datos proporcionados por la encuesta sobre los usos del agua del 2014 , las redes públicas abastecieron a las ciudades con $4.873 \mathrm{hm}^{3}$ de agua, donde cerca de un $18 \%$ se pierde por averías, errores de medida o fraude. En el 2015, la distribución alcanzó los $5.379 \mathrm{hm}^{3}$, incrementándose el porcentaje en un $21 \%$, a pesar de que consumo de hogares y empresas descendió, el resultado final es un importante incremento del volumen total de agua distribuida. De su análisis e interpretación se coligen las siguientes cuestiones:

3 Sotelo Navalpotro, J. A. et alii (2010) en «La «Huella Hídrica» española en el contexto del cambio ambiental», es el primer caso en nuestro país, que se desciende a nivel infraestatal en la estimación de la «Huella Hídrica», llegando hasta el nivel municipal.

${ }^{4}$ Por ejemplo, el agua de riego de jardines, y de policía urbana, no está contabilizada; al igual que el agua utilizada en el sector de la construcción, el llamado agua de «obra», también deja fuera de contabilidad muchos metros cúbicos, que no son facturados, o al menos no lo son en la misma medida que el agua domestica, industrial o de empresas de servicios. 
a) El total de agua consumida y distribuida por empresas y hogares apenas llega al $15 \%$ de la que se demanda en el sector agrario para el regadío, por lo que las mayores cantidades de agua no son utilizadas en las ciudades.

b) Un porcentaje muy elevado del agua potable distribuida no se contabiliza por pérdidas, errores de medidas o fugas

c) El agua distribuida por redes públicas de suministro es solo una parte de los recursos hídricos necesarios para sostener el conjunto de las actividades humanas, y por lo tanto, la única mejor cuantificada, a pesar de sus abultados errores.

Con todo ello, y atendiendo a la metodología de cálculo establecida en el presente estudio, podemos observar como la composición del consumo de agua en las ciudades de España vienen determinada, a nivel global y regional, por el agua distribuida a los hogares (representa el 54,56\% sobre el total), y por las actividades económicas desarrolladas en las urbes (con un 17,16\%), si bien, la suma del volumen total de pérdidas de recursos hídricos en el proceso de distribución y el agua distribuida no registrada (20,44\%) es, cuanto menos, excesivo pues supera con creces al total de agua empleada en el desarrollo de la propia actividad económica así como los consumos municipales (ver tabla 1).

TABLA 1

CONSUMO DE AGUA EN LAS CIUDADES ESPAÑOLAS (2015)

\begin{tabular}{l|r|r}
\multicolumn{1}{c|}{ Agua Distribuida } & \multicolumn{1}{c}{$\mathbf{h m}^{3}$} & \% sobre Total \\
\hline Hogares & 2.935 & $54,56 \%$ \\
\hline Actividades Económicas & 923 & $17,16 \%$ \\
\hline Consumos Municipales y otros & 422 & $7,85 \%$ \\
\hline Pérdidas & 720 & $13,39 \%$ \\
\hline Agua distribuida no registrada & 379 & $7,05 \%$ \\
\hline Total & 5.379 & $100 \%$ \\
\hline Agua de Regadío & 16.927 & \\
\hline
\end{tabular}

Fuente: Elaboración propia. 
FIGURA 1

EXTRACCIONES DE AGUA POR SECTORES ECONÓMICOS (2015)
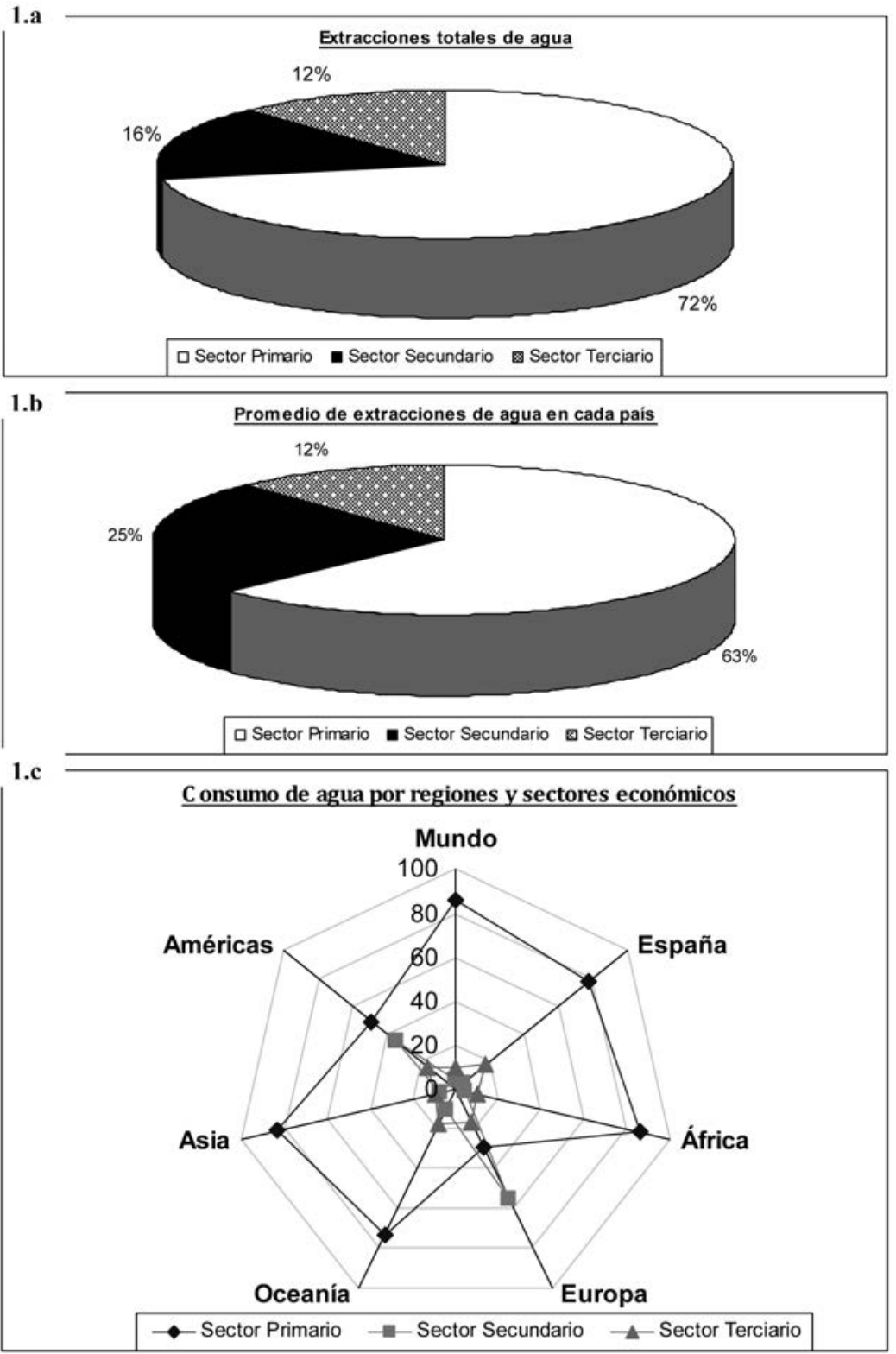

Fuente: Elaboración propia.

Estudios Geográficos, Vol. LXXIX, 284, pp. 115-140, enero-junio 2018

ISSN: 0014-1496, eISSN: 1988-8546, doi: https//doi.org/10.3989/estgeogr.201805 
En cuanto a las extracciones de recursos hídricos en relación con las áreas urbanas, podemos observar como el sector primario es el que demanda mayores cantidades de agua - concretamente, el sector de la agricultura es el principal demandante de agua - , seguido del sector industrial (16\%), dejando en último lugar al sector servicios, por lo que se puede deducir que el porcentaje total de extracciones necesarios en las ciudades son menores que en otros territorios, lo que no quiere decir, como veremos a continuación, que demande menores cantidades de «Huella Hídrica» (ver figura 1.a).

De igual modo, existe una gran variedad regional del destino final del volumen total de agua extraída en los grandes sectores económicos, estableciendo notables diferencias territoriales, concretamente en las grandes ciudades españolas. Así pues, se puede establecer o distinguir diversos modelos, entre los que destacan, el que podríamos denominar «modelo africano», caracterizado por el enorme sobrepeso porcentual del consumo agropecuario, y un valor muy bajo del correspondiente a actividades industriales (ver figura 1.b).

En nuestro caso, cobra especial interés el denominado modelo europeo, pudiéndose afirmar que los valores entre la Europa Occidental y Central, son prácticamente iguales y corresponden a los del perfiles globales de Europa, pero si descendemos a niveles regionales inferiores las diferencias se hacen patentes y acusadas entre los del Norte, Oeste, Central y Mediterránea, como consecuencia, entre otras cuestiones, a la diversidad física, climática y a sus condiciones intrínsecas de sus propios medios naturales, así como a sus estructuras económicas (la región mediterránea europea se aleja del perfil general, tanto en consumo industrial de agua, como en el agropecuario, el primero por defecto, el segundo por exceso) (ver figura 1.c).

En el caso concreto de España, tiene un modelo de consumo de recursos hídricos europeo en cuanto a demandas de agua urbanas, y africano en el sector agropecuario e industrial. Esto es consecuencia directa de la pérdida de peso de la actividad industrial en "pro» del sector servicios, así mismo, la importancia del regadío en la región mediterránea, y, más concretamente en España, puede explicar la «mixticidad» de este perfil de consumo (ver tabla 2).

Del mismo modo, debemos tener presente que no todas las actividades de servicios e industriales se generan en las ciudades, pero si se dan en ellas la mayor concentración de las mismas, por lo que adjudicar a las ciudades todo el caudal hídrico que consume industria y servicios no sería del todo realista. 
TABLA 2

PORCENTAJE DE EXTRACCIÓN DE AGUA POR SECTORES ECONÓMICOS (2015)

\begin{tabular}{|c|c|c|c|}
\hline & $\begin{array}{c}\text { Sector } \\
\text { Primario (\%) }\end{array}$ & $\begin{array}{c}\text { Sector } \\
\text { Secundario (\%) }\end{array}$ & $\begin{array}{c}\text { Sector } \\
\text { Terciario (\%) }\end{array}$ \\
\hline TOTAL EN EL MUNDO & 72 & 16 & 12 \\
\hline ÁFRICA & 85 & 5 & 10 \\
\hline Septentrional & 86 & 4 & 10 \\
\hline Subsahariana & 87 & 2 & 11 \\
\hline AMÉRICA & 50 & 32 & 18 \\
\hline Septentrional & 43 & 43 & 15 \\
\hline Central y Caribe & 65 & 10 & 26 \\
\hline Sur & 68 & 13 & 19 \\
\hline ASIA & 85 & 6 & 8 \\
\hline OCEANÍA & 73 & 10 & 17 \\
\hline EUROPA & 29 & 55 & 16 \\
\hline Europa Occidental y Central & 28 & 56 & 16 \\
\hline Norte & 12 & 61 & 26 \\
\hline Oeste & 8 & 77 & 16 \\
\hline Central & 8 & 78 & 14 \\
\hline Mediterránea & 61 & 24 & 16 \\
\hline España $(*)$ & 75 & 7 & 18 \\
\hline Europa oriental & 32 & 51 & 18 \\
\hline Oriental & 49 & 37 & 14 \\
\hline Fed. Rusa & 20 & 60 & 20 \\
\hline
\end{tabular}

Fuente: Elaboración propia.

Podemos afirmar, no obstante, que las ciudades son consumidoras netas de «Agua Virtual», como consecuencia directa del nivel total de población que, en el caso concreto de nuestro país, representa un setenta por ciento del total de población nacional. Con todo ello, reiterar que las ciudades son los territorios que menos recursos hídricos consumen, desde el punto de vista "per cápita», a pesar de presentar los mayores consumos de «Agua Virtual». 
Por otra parte señalar que en nuestra investigación estudiamos las ciudades españolas, escogidas como principales en la base de datos del Eurostat, mostrando un comportamiento muy desigual en el consumo doméstico «per cápita» del agua, el cual está relacionado con los niveles de población, y por tanto, vinculado al tamaño de ésta (ver figura 2). Sin embargo, no existe una correlación perfecta entre el volumen de población y el gasto «per cápita» de agua.

\section{FIGURA 2}

\section{CONSUMO DE RECURSOS HÍDRICOS EN LAS PRINCIPALES CIUDADES ESPAÑOLAS (2015)}

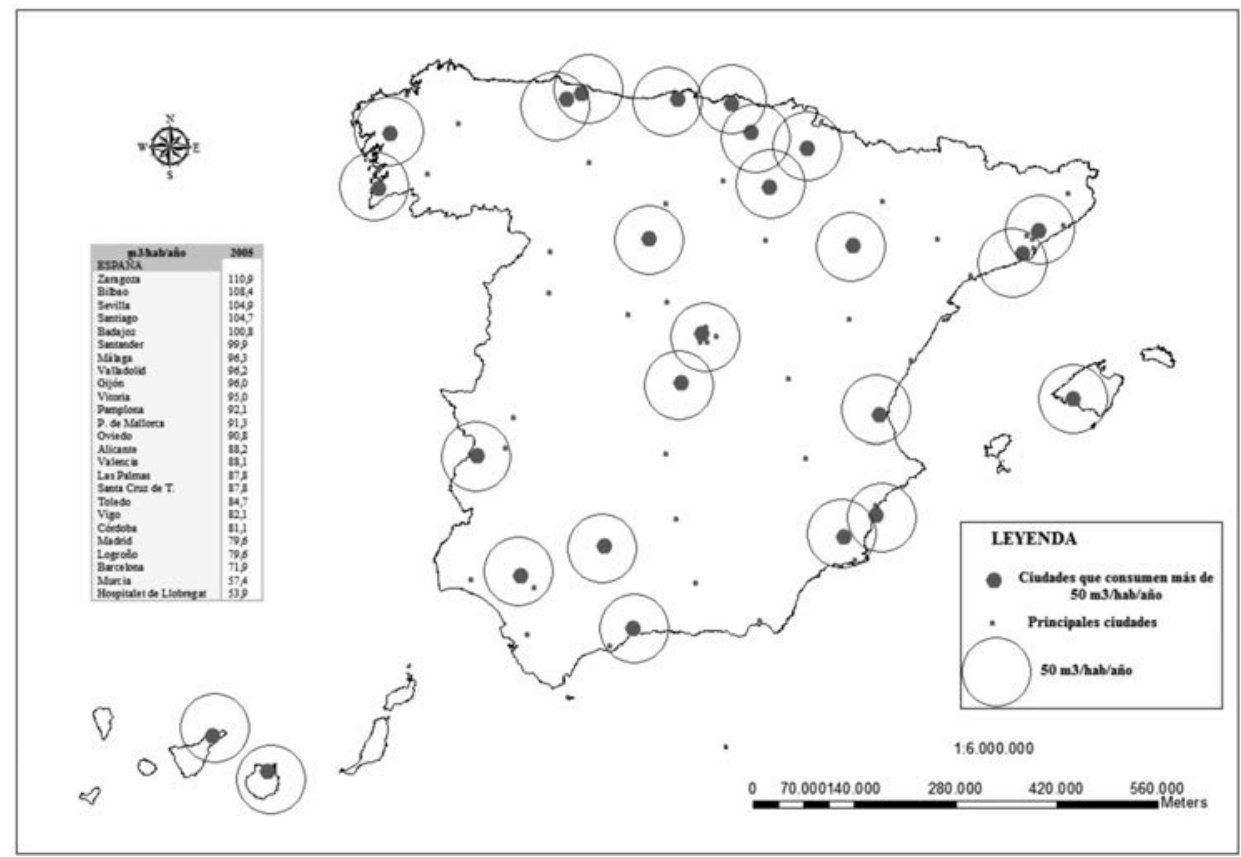

Fuente: Elaboración propia, a partir de gasto «Urban Indicator for Core Cities» (Eurostat).

Igualmente, cabe señalar que las condiciones naturales —climáticas y físicas - tampoco repercuten de manera estrictamente directa en las demandas o consumos de agua; el consumo de recursos hídricos. Y es que la desigualdad del patrón de consumo en nuestro medio urbano no está relacionado ni con las condiciones climáticas ni con el modelo de ciudad, aunque, podríamos pensar que esto es consecuencia de que la estructura de los sistemas metropolitanos es similar en toda España. 
Por otra parte, si procedemos al análisis detallado de las demandas de recursos hídricos, observamos como Zaragoza, Sevilla, Santiago de Compostela, Bilbao y Badajoz son las ciudades que consumen mayores cantidades de agua -lo que nos muestra una gran heterogeneidad geográfica, por sus diferencias físicas, climáticas, económicas, demográficas, sociales y estructurales-. En contraposición, destacan grandes urbes como Madrid y Barcelona, las cuales presentan los menores niveles de consumo de agua, «per cápita».

La única variable con la que el consumo parece tener una cierta relación, aunque tampoco resulta determinante, es el precio, pues, con valor unitario, la facturación final resulta más abultada por otros conceptos que por el mismo precio del metro cúbico servido. Pese a ello, las regiones en las que existe una gran oferta de recursos hídricos y el precio es bajo, los consumos de agua, en general, y de «Huella Hídrica», en particular, se incrementan. Otro aspecto interesante a tener en cuenta es el diferencial del consumo de estas ciudades con relación a sus respectivos territorios en los que se enmarcan. Salvo el caso de Murcia, donde el consumo "per cápita» urbano está ligeramente por debajo del de su Comunidad Autónoma, en el conjunto de las ciudades tratadas, éste es superior, aunque el diferencial es altamente variable, pues el cociente entre el consumo "per cápita» de la ciudad y su Comunidad Autónoma correspondiente varia entre el 1,9 en Zaragoza, y el 0,97 en Murcia.

Con todo ello, podríamos pensar que en las ciudades se demandan y consumen mayores niveles de recursos hídricos, pero debemos tener en cuenta que los valores expuestos solo responden a los niveles de agua suministrada por la red pública de abastecimiento y, hoy por hoy, en las ciudades el agua está mejor medida que en otros territorios. Igualmente, debemos tener presente que estos patrones de consumo se modifican cuando analizamos las demandas de «Huella Hídrica» - y no solo tenemos en cuenta el consumo directo de agua (ver tabla 3 ).

Por otra parte, en lo que al consumo de agua de las grandes ciudades españolas se refiere, nos muestra que existe una considerable desigualdad entre las distintas Comunidades Autónomas. Ciudades como Madrid, Barcelona y Valencia, están muy próximos a los consumos de su territorio regional; sin embargo, ciudades como Zaragoza, Vitoria, Bilbao, Valladolid, Palma de Mallorca o Sevilla, presentan niveles superiores al consumo de sus territorios de influencia. Por su parte, en el País Vasco, Vitoria y Bilbao; en Cataluña, Barcelona y Hospitalet de Llobregat, están al mismo nivel de sobreconsumo respecto a su región, mientras que en el País Valenciano, Alicante y Valencia están en cabeza, y, en Asturias, Gijón y Oviedo al final de las mismas. A su vez, señalar que el conjunto de estas ciudades demandan el veinticinco por 
TABLA 3

CONSUMO DE AGUA POR COMUNIDADES AUTÓNOMAS Y GRANDES CIUDADES ESPAÑOLAS (2015)

\begin{tabular}{|c|c|c|c|c|}
\hline & Ciudad & C.Autónoma & Comunidad & Cociente \\
\hline & $\mathrm{m}^{3} / \mathrm{hab} / \mathrm{Año}$ & $\mathrm{m}^{3} / \mathrm{hab} / \mathrm{Año}$ & Autónoma & Ciudad/CA \\
\hline Zaragoza & 110,9 & 58,035 & Aragón & 1,91 \\
\hline Vitoria & 95 & 50,735 & País vasco & 1,87 \\
\hline Bilbao & 108,4 & 59,13 & País vasco & 1,83 \\
\hline Valladolid & 96,2 & 52,925 & Castilla y León & 1,82 \\
\hline Badajoz & 100,8 & 55,845 & Extremadura & 1,8 \\
\hline Alicante & 88,2 & 48,91 & Com. Valenciana & 1,8 \\
\hline Palma de Mallorca & 91,3 & 51,1 & Baleares & 1,79 \\
\hline Sevilla & 104,9 & 62,415 & Andalucía & 1,68 \\
\hline Pamplona & 92,1 & 55,48 & Navarra & 1,66 \\
\hline Gijón & 96 & 58,4 & Asturias & 1,64 \\
\hline Málaga & 96,3 & 59,13 & Andalucía & 1,63 \\
\hline Vigo & 82,1 & 51,1 & Galicia & 1,61 \\
\hline Toledo & 84,7 & 52,925 & C. Mancha & 1,6 \\
\hline Santiago & 104,7 & 71,175 & Galicia & 1,47 \\
\hline Logroño & 79,6 & 55,48 & Rioja & 1,43 \\
\hline Santander & 99,9 & 71,175 & Cantabria & 1,4 \\
\hline Santa Cruz de Tenerife & 87,8 & 63,145 & Canarias & 1,39 \\
\hline Las palmas & 87,8 & 63,51 & Canarias & 1,38 \\
\hline Oviedo & 90,8 & 65,7 & Asturias & 1,38 \\
\hline Madrid & 79,6 & 62,415 & Com. Madrid & 1,28 \\
\hline Valencia & 88,1 & 69,715 & Com. Valenciana & 1,26 \\
\hline Córdoba & 81,1 & 71,175 & Andalucía & 1,14 \\
\hline Barcelona & 71,9 & 65,7 & Cataluña & 1,09 \\
\hline Hospitalet de Llobregat & 53,9 & 52,925 & Cataluña & 1,02 \\
\hline Murcia & 57,4 & 59,13 & C. Murcia & 0,97 \\
\hline
\end{tabular}

Fuente: Elaboración propia, a través de la Base de datos de Aquastat-FAO y la Encuesta sobre Suministros y Tratamiento del Agua (INE, 2016). 
ciento del total de agua doméstica suministrada por las redes públicas de abastecimiento, con lo que la teoría del crecimiento endógeno ha dado un paso adelante para entender el comportamiento de la productividad, ya que considera que los rendimientos decrecientes son tan solo uno de los resultados posibles del funcionamiento del proceso de acumulación de capital. Si bien, existen otras vías de crecimiento económico cuando las inversiones en bienes de capital, incluido el capital humano, generan rendimientos crecientes, como consecuencia de la difusión de las innovaciones y del conocimiento entre las empresas y la creación de economías externas (García Alvarado, J.M., 2000). La interpretación del desarrollo endógeno, o si se prefiere la visión territorial del desarrollo, a su vez, se caracteriza por rasgos muy específico, que Boisier (2003) sintetiza al conceptualizar lo que el denomina la «endogeneidad».

Segundos Resultados. las ciudades españolas y Su «Huella Hídrica»

Llegados a este punto, pasamos a evaluar el peso que tienen las ciudades en el conjunto del consumo hídrico, una vez incorporado el «Agua Virtual», empleando como base el conjunto de ciudades establecidas por el «core cities in Spain», a efectos de indicadores ambientales, los datos ofrecidos por la publicación «La Huella Hídrica española en el contexto del cambio climático» (Sotelo Navalpotro, J.A, 2010), así como la propia elaboración de las bases de datos.

El estudio de la relación existente entre el agua doméstica suministrada y el «Agua Virtual» empleada en la producción vs. consumo de bienes y servicios, en las urbes españolas, nos muestra que las ciudades seleccionadas, sólo siete de ellas están por debajo de la «Huella Hídrica per cápita» nacional, y, a pesar de ello, sus valores se encuentran próximos a la media del país — por lo que, aunque nos estemos moviendo sólo con una muestra, podemos afirmar que las ciudades demandan una parte muy importante de la «Huella Hídrica» del país (ver tabla 4).

En cuanto al agua de consumo, se observa como, con el valor de la «Huella Hídrica» en la jerarquía urbana y teniendo en cuenta el tamaño medio de la vivienda, presentan un coeficiente de correlación relativamente débil, por lo que no parece que el habitante de una gran ciudad consuma sensiblemente más recursos hídricos que el de una ciudad de tipo medio o pequeño. Por el contrario, la correlación densidad de población, consumo "per cápita» es inversa, lo que podría dar a entender un hecho, y es que las aglomeraciones más densas son más 
TABLA 4

LA «HUELLA HÍDRICA PER CÁPITA» EN LAS CIUDADES ESPAÑOLAS (2015)

\begin{tabular}{|c|c|c|c|c|}
\hline & Población & $\mathrm{hm}^{3}$ & $\mathrm{~m}^{3} /$ per cápita & España/municipio \\
\hline ESPAÑA & 46.438 .422 & & 2611,31 & \\
\hline Madrid & 3.099 .834 & 8125,05 & 2621,1 & 1,13 \\
\hline Barcelona & 1.578 .546 & 4108,54 & 2602,7 & 1,12 \\
\hline Valencia & 785.732 & 1806,57 & 2299,2 & 0,99 \\
\hline Sevilla & 704.203 & 1684,34 & 2391,8 & 1,03 \\
\hline Zaragoza & 638.799 & 1372,43 & 2148,5 & 0,92 \\
\hline Málaga & 547.731 & 1335,42 & 2438,1 & 1,05 \\
\hline Murcia & 398.815 & 1020,02 & 2557,6 & 1,1 \\
\hline Las Palmas & 376.953 & 798,91 & 2119,4 & 0,91 \\
\hline Valladolid & 321.713 & 740,87 & 2302,9 & 0,99 \\
\hline Palma de Mallorca & 368.974 & 863,53 & 2340,4 & 1,01 \\
\hline Santiago & 92.298 & 217,43 & 2355,7 & 1,01 \\
\hline Vitoria & 223.702 & 586,16 & 2620,3 & 1,13 \\
\hline Oviedo & 209.495 & 472,72 & 2256,5 & 0,97 \\
\hline Pamplona & 191.865 & 515,03 & 2684,3 & 1,15 \\
\hline Santander & 183.799 & 429,35 & 2336 & 1 \\
\hline Toledo & 73.485 & 180,67 & 2458,6 & 1,06 \\
\hline Badajoz & 139.135 & 336,82 & 2420,8 & 1,04 \\
\hline Logroño & 141.568 & 329 & 2324 & 1 \\
\hline Bilbao & 352.317 & 914,01 & 2594,3 & 1,12 \\
\hline Córdoba & 314.178 & 768,22 & 2445,2 & 1,05 \\
\hline Alicante & 310.330 & 724,35 & 2334,1 & 1 \\
\hline Vigo & 292.059 & 687,32 & 2353,4 & 1,01 \\
\hline Gijón & 271.039 & 610,32 & 2251,8 & 0,97 \\
\hline Hospitalet de Llobregat & 250.536 & 652,19 & 2603,2 & 1,12 \\
\hline Santa Cruz de Tenerife & 219.446 & 471,51 & 2148,6 & 0,92 \\
\hline SUMA O PROMEDIO & 12086552 & 29750,78 & 2400,3 & 1 \\
\hline
\end{tabular}

Fuente: Elaboración propia. 
eficientes en el uso del agua potable; sin embargo, el tamaño medio de vivienda parece no tener mucha relación con el consumo de agua de "suministro», aunque, si presenta relación con dos de los aspectos contemplados, a saber, jerarquía urbana, y «Agua Virtual» vs. «Huella Hídrica». En el primero de los casos la relación es inversa, a mayor jerarquía urbana menor tamaño medio de superficie de vivienda; esto tiene su lógica, en tanto que las ciudades mayores tienen una mayor presión sobre suelo y vivienda, que las de rango inferior. Por otro lado, a mayor superficie media, mayor consumo de agua virtual; el valor negativo en los casos en los que se correlaciona la jerarquía, obedece a que el rango se ha tabulado de 1 a 5 , siendo 1 el mayor puesto en la escala y 5 el menor. Mientras que el valor «per cápita» del consumo urbano muestra una muy pequeña correlación con el volumen precipitaciones medias anuales (INM serie 1971-2000) (sin lugar a duda este consumo puede estar influido, por otras variables climáticas, pero desde luego, no lo parece estar significativamente, con relación al volumen de precipitaciones) (ver tabla 5).

\section{TABLA 5}

AGUA Y CIUDAD: COEFICIENTE DE CORRELACIÓN

\begin{tabular}{l|c|c}
\hline Agua Virtual-Agua Doméstica & 0,188 & \\
\hline Agua Doméstica-Jerarquía Urbana & & 0,05 \\
\hline Agua Virtual-Jerarquía Urbana & & \\
\hline $\mathrm{m}^{2}$ vivienda-Agua Virtual & 0,673 & \\
\hline $\mathrm{m}^{2}$ vivienda-Agua Doméstica & & 0,405 \\
\hline $\mathrm{m}^{2}$ vivienda-Jerarquíca & & 0,075 \\
\hline & & 0,405 \\
\hline Hab/Km ${ }^{2}$-Jerarquía & 0,535 & \\
\hline Hab/Km ${ }^{2}$-Agua Doméstica & & - \\
\hline Precio-Jerarquía & 0,435 & \\
\hline Población-Jerarquía & & - \\
\hline Km2-Agua Virtual & 0,139 & \\
\hline Consumo-precipitaciones en mm & & \\
\hline Población/«Huella Hídrica» per cápita & 0,658 & \\
\hline
\end{tabular}

Fuente: Elaboración propia. 
Del mismo modo, el promedio de «Huella Hídrica per cápita» en la muestra urbana demanda un total de $2.400 \mathrm{~m}^{3} / \mathrm{hab} /$ año para unos 480.000 habitantes, y de ello se deduce que este módulo demográfico no tiene una relación plena con la «Huella Hídrica» expresada en su forma «per cápita». Claro ejemplo de ello lo encontramos en el caso concreto de Madrid, donde la población es 6,5 veces la del promedio, por lo que la «Huella Hídrica» debería ser de $17.036 \mathrm{~m}^{3} /$ hab/año, frente a la de 2.621 obtenida tras los cálculos. De igual modo sucede en Barcelona, en la que su «Huella Hídrica» debería ser de $8.499 \mathrm{~m}^{3} / \mathrm{hab} / \mathrm{año}$ y ésta apenas supera los $2.600 \mathrm{~m}^{3} / \mathrm{hab} / \mathrm{año}$. Se demuestra así que la naturaleza de gran ciudad no conlleva en proporción un mayor derroche de recursos hídricos, de hecho, en muchos casos, ocurre lo contrario, se lleva a cabo un uso más eficiente de los mismos (si bien, los niveles de "Huella Hídrica» varían o se modifican si realizamos un estudio pormenorizado de los llamados colores de «Huella Hídrica» — aunque, no incide de manera significativa en los resultados obtenidos).

FIGURA 3

«HUELLA HÍDRICA» Y JERARQUÍA URBANA EN ESPAÑA

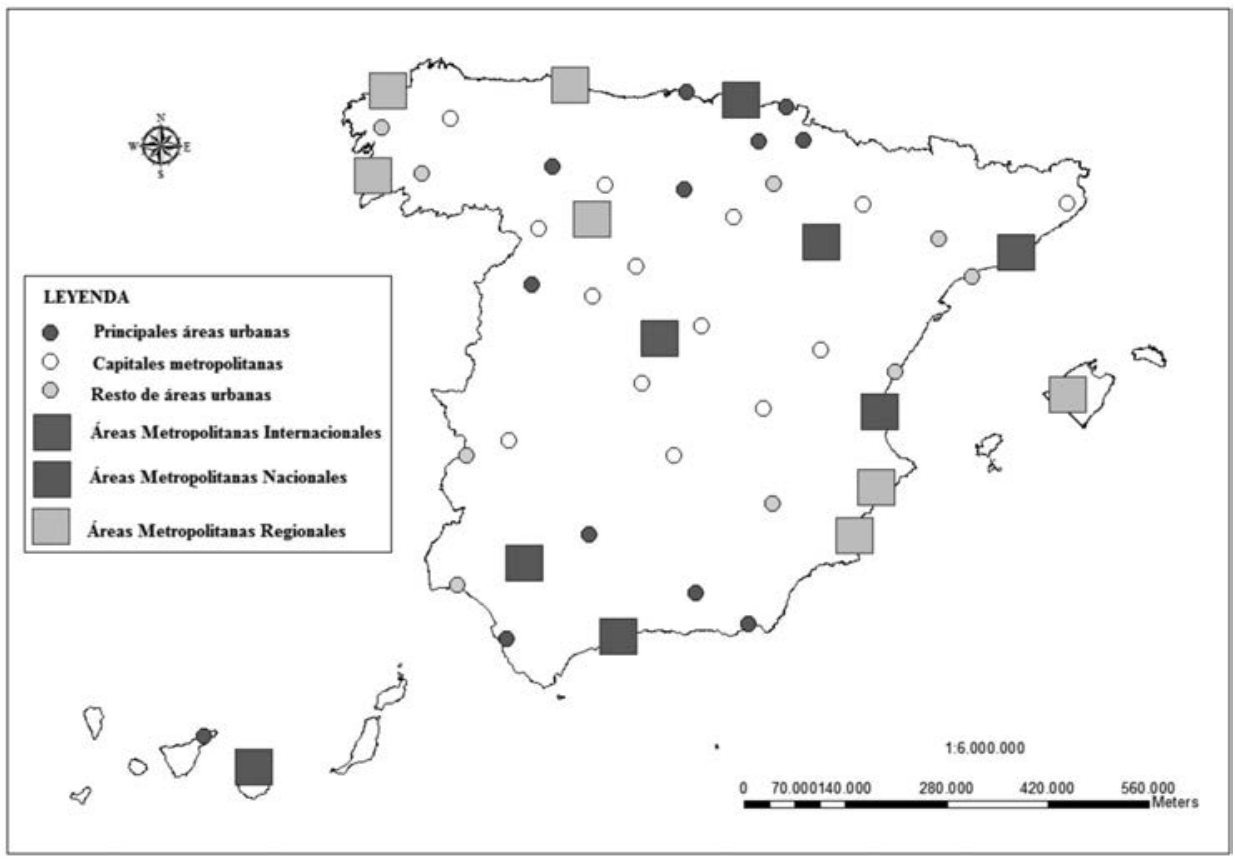

Fuente: Elaboración propia, a partir de gasto «Urban Indicator for Core Cities» (Eurostat). 
Por otra parte, si nos detenemos en la propia jerarquía urbana (ver figura 3), podemos diferenciar tres tipos de niveles; en el primero de ellos se nos muestran ciudades como Madrid y Barcelona que, dada su condición de centralidad nacional, tienen un posicionamiento y funciones internacionales que permite consideradas como metrópolis nacionales de rango internacional, y en puestos de cabeza del sistema urbano europeo. Estas son las que presentan un mayor consumo de «Huella Hídrica». En ambos casos se trata de municipios centrales de unas áreas urbanas metropolitanas cuyo tamaño supera el millón de personas y, cuyo perfil corresponde con espacios urbanos consolidados -en términos económicos, sociales, territoriales,...- cuya reciente expansión obedecen al modelo de la ciudad post-industrial, meta-industrial o de servicios y, cuya realidad demográfica es mayor que la que aquí se ha considerado al restringirnos, por exigencia de fuente, solo al municipio principal. Todo ello sin olvidarnos que, a nivel provincial, tanto Madrid como Barcelona suponen más del treinta por ciento del Producto Interior Bruto nacional — con lo que cada hm $3 /$ año, genera en Madrid 16,4euros, y en Barcelona, 19,6 euros del total del PIB-; con lo que, en cualquier caso, esta extrapolación del valor provincial no tiene gran valor de aproximación, salvo en aquellos casos de provincias presididas por una gran y única ciudad.

Un segundo nivel está formado por las ciudades que ostenta una centralidad regional, pero con funciones de alcance nacional. Esto es, se tratan de ciudades cabeceras de regiones, pero cuyo perfil actual supera el de las propias regiones. Su dinámica y el alcance de sus relaciones rebasa con creces al de su propio territorio, ocupando un lugar destacado en nuestro sistema urbano nacional. Se trata de Valencia, Sevilla, Zaragoza, Málaga, Las Palmas y Bilbao, y con un rango que oscila entre los 360.000 y los 800.000 habitantes. Es cierto que en este nivel hay diferencias importantes de tamaño, pues va desde 1 a casi 3 la variabilidad del tamaño; es bien conocido por la geografía urbana, que, ni las características, ni las llamadas "patologías urbanas» de una ciudad de 1000.000 de habitantes son el simple sumatorio de tres ciudades 300.000, ¿ocurrirá lo mismo con la «Huella Hídrica»?

Desde esta perspectiva, con relación al grupo anterior se produce un descenso significativo de la «Huella Hídrica». Como no podía ser de otro modo, el valor de la «Huella Hídrica» de estas ciudades se encuentra a mucha distancia de las de Madrid o de Barcelona, pero esto solo es un efecto del diferencial de población. Si nos detenemos en el valor per cápita, hay un descenso de la huella, pero no en la proporción en que lo hay de población (nótese como Bilbao, tiene una huella casi similar a la de Barcelona; es de destacar el caso de Zaragoza y Las Palmas, especialmente la primera, que con unas poblaciones muy desiguales, la huella es muy similar). 
En las otras categorías, se produce un decrecimiento de la «Huella Hídrica» en cierta consonancia con el descenso de la categoría urbana. Llama la atención el caso de Murcia, que presenta un valor bastante elevado, en relación con el conjunto de ciudades de su rango. Una posible explicación la encontramos en la importancia que tiene, respecto de la «Huella Hídrica», en su Área Metropolitana el sector hostelero, el comercio, el transporte, los servicios privados y sociales, y la industria, o bien errores en las «fuentes» estadísticas.

Las ciudades son las principales protagonistas de la translimitación hídrica de nuestro país y causantes de una importante «Huella Hídrica». No debemos olvidarnos de dos aspectos muy importantes, a saber: el nivel de eficiencia, y el valor del agua virtual transferida. Sin embargo, el segundo de los aspectos es, prácticamente, hoy por hoy, de un cálculo imposible. En una economía exportadora de bienes de base agropecuaria, e incluso industrial de base, hay ciertas posibilidades de atribuir a esa producción exportada, una «Huella Hídrica», pues la mayoría de las metodologías de cálculo se están estableciendo en esas ramas de actividad. Sin embargo no parece existir, todavía, un cálculo de ese mismo valor para la producción - exportacióndel sector servicios, preponderante en los espacios urbanos, y especialmente los productos derivados de servicios avanzados. Las ciudades estudiadas, son tan solo una parte, de las ciudades españolas, cuyo umbral de selección no ha sido estrictamente el tamaño de población, ya que hay ciudades mayores de 100.000 habitantes que no aparecen, y otras menores que si lo hacen en tanto tienen carácter de capitalidad administrativa.

Ante la falta de datos macroeconómicos desagregados a nivel municipal, hemos optado por hacer uso del indicador llamado «Índice de Actividad Económica», en adelante (IAE). Ese se establece con base en el impuesto correspondiente al total de actividades económicas empresariales (industriales, comerciales y de servicios) y profesionales, a excepción de las agrarias, las cuales no están sujetas al impuesto de actividades económicas.

En nuestro estudio se demuestra que hay una correlación importante entre el valor del IAE y «Huella Hídrica» anual per cápita ( $(\mathrm{2} 2=0,944, \mathrm{r}=0,972)$, en la serie de las ciudades consideradas (ver tabla 6).

La serie de ciudades estudiadas aportan más del $46 \%$ de la actividad económica que refleja el índice total del país, por lo que si bien algunas de estas ciudades superan con creces la media de «Huella Hídrica» per cápita nacional, la mayoría de ellas se encuentran muy por debajo de este valor.

Una cuestión es si ese consumo de agua virtual tiene una correspondencia justa con la intensidad de la vida económica, término al que podríamos llamar 
la «eficiencia económica» de los diferentes consumos de agua virtual (es decir, cuánto cuesta hídricamente la obtención de los diferentes valores del indicador de actividad económica).

TABLA 6

«HUELLA HÍDRICA» Y ACTIVIDAD ECONÓMICA EN ESPAÑA (2015)

\begin{tabular}{l|c|c|c|c}
\hline & Habitantes & IAE & H.H. & E.E.H.H. \\
\hline ESPANA & 46.438 .422 & 100.000 & 101.434 & 1,00 \\
\hline Barcelona & 1578546 & 9.196 & 4108,54 & 2,24 \\
\hline Pamplona & 191865 & 1.121 & 515,03 & 2,18 \\
\hline Valencia & 785732 & 3.629 & 1806,57 & 2,01 \\
\hline Oviedo & 209495 & 872 & 472,72 & 1,84 \\
\hline Santiago de Compostela & 92298 & 401 & 217,43 & 1,84 \\
\hline Las palmas & 376953 & 1.326 & 798,91 & 1,66 \\
\hline Palma de Mallorca & 368974 & 1.430 & 863,53 & 1,66 \\
\hline Bilbao & 352317 & 1.478 & 914,01 & 1,62 \\
\hline Santa Cruz de Tenerife & 219446 & 758 & 471,51 & 1,61 \\
\hline Sevilla & 704203 & 2.632 & 1684,34 & 1,56 \\
\hline Santander & 183799 & 661 & 429,35 & 1,54 \\
\hline Madrid & 3099834 & 12.020 & 8125,05 & 1,48 \\
\hline Zaragoza & 638799 & 1.953 & 1372,43 & 1,42 \\
\hline Alicante & 310330 & 1.021 & 724,35 & 1,41 \\
\hline Toledo & 73485 & 253 & 180,67 & 1,4 \\
\hline Logroño & 141568 & 440 & 329 & 1,34 \\
\hline Valladolid & 321713 & 960 & 740,87 & 1,3 \\
\hline Córdoba & 314178 & 897 & 768,22 & 1,17 \\
\hline Badajoz & 139135 & 392 & 336,82 & 1,16 \\
\hline Málaga & 547731 & 1.509 & 1335,42 & 1,13 \\
\hline Vigo & 292059 & 775 & 687,32 & 1,13 \\
\hline Murcia & 398815 & 1.099 & 1020,02 & 1,08 \\
\hline Gijón & 271039 & 638 & 610,32 & 1,05 \\
\hline Vitoria & 223702 & 496 & 586,16 & 0,85 \\
\hline Promedios-Sumas & & 46.231 & 29750,8 & 1,4272 \\
\hline Coef. Correlación & IAE-Huella H. & & 0,972 & \\
\hline & & & & \\
\hline
\end{tabular}

Fuente: Elaboración propia. 
Para conseguir un valor de $46 \%$ de la actividad, la «Huella Hídrica» es de $29750,8 \mathrm{hm}^{3} / \mathrm{año}$; o lo que es lo mismo, que cada $\mathrm{hm}^{3}$, genera en el conjunto de ciudades 1,55 puntos, frente al 1 del conjunto del país. En ese sentido, las ciudades más eficientes en el balance actividad económica - «Huella Hídrica», serían Barcelona, Pamplona y Valencia, las cuales duplicarían la eficacia hídrica de la media nacional.

De esta forma, con el objeto de lograr un desarrollo sostenible, y mitigación de la translimitación de huella ecológica urbana, deberían plantearse objetivos de incremento del valor de la actividad, con el decrecimiento del valor de la huella, esto es conseguir una mayor eficiencia del sistema productivo urbano en cuanto a recurso hídrico, propio e importado se refiere.

Puede afirmarse que tampoco hay un comportamiento uniforme entre las ciudades, es más, puede verse como se deshace ese comportamiento gemelo de las dos grandes ciudades como Barcelona y Madrid, al incluir esta variable nueva. Efectivamente, Barcelona muestra como una mayor eficacia productiva en cuanto a ese consumo, frente a Madrid, que se halla 0,48 puntos por encima del valor medio.

Es de señalar, como el valor de Madrid, 1,48, parte en dos grupos casi de igual número, por arriba y por debajo, predominando en el grupo más eficiente las ciudades mayores, y en el grupo de mayores causantes de «huella» las de menor tamaño y categoría en relación con su actividad económica, con puntuales excepciones en uno y otro grupo. En este sentido se vuelve a revelar que las mayores aglomeraciones, si bien son causantes, en proporción de mayor gasto de recursos hídricos, respecto a la «huella», al «agua virtual», son las de mayor eficiencia económica. Eficiencia que se ve matizada por la «Huella Hídrica» de la eficiencia de la red de abastecimiento de las ciudades de nuestro país (ver tabla 7).

Las pérdidas físicas de la red son la parte más importante de la denominada «Agua No Registrada», que mete en el mismo cajón los consumos no autorizados (fraudes), los consumos autorizados no medidos, los errores de medida y las fugas de la red. La media de este indicador en España ronda el 25\%, que es lo mismo que decir que la cuarta parte del agua urbana suministrada se desperdicia, aunque en el 2015, se redujo al 23\% gracias — según intuyen los operadores- a la tecnificación del sector, que cada vez dispone de mejores sistemas para detectar las fugas y contenerlas ${ }^{5}$.

5 AEAS (2016). «XIV Estudio Nacional de Suministro de Agua Potable y Saneamiento en España, 2016». 


\section{TABLA 7}

LA «HUELLA HÍDRICA» DE LA EFICIENCIA DE LA RED DE ABASTECIMIENTO EN LAS CIUDADES ESPAÑOLAS (2015)

\begin{tabular}{|c|c|c|}
\hline & $\begin{array}{l}\text { Huella Hídrica }\left(\mathrm{hm}^{3}\right) \\
\text { Eficiencia red de } \\
\text { abastecimiento }\end{array}$ & $\begin{array}{l}\text { Porcentaje de Huella Hídrica } \\
\text { respecto a la eficiencia de la } \\
\text { red de agua potable (\%) }\end{array}$ \\
\hline Madrid & 889,74 & 7,55 \\
\hline Barcelona & 449,91 & 8,16 \\
\hline Valencia & 197,83 & 16,14 \\
\hline Sevilla & 184,44 & 21,92 \\
\hline Zaragoza & 150,29 & 32,47 \\
\hline Málaga & 146,24 & 23,91 \\
\hline Murcia & 111,70 & 25,84 \\
\hline Las Palmas & 87,48 & 51,12 \\
\hline Valladolid & 81,13 & 19,99 \\
\hline Palma de Mallorca & 94,56 & 24,30 \\
\hline Santiago & 23,81 & 31,20 \\
\hline Vitoria & 64,19 & 12,15 \\
\hline Oviedo & 51,77 & 28,21 \\
\hline Pamplona & 56,40 & 12,05 \\
\hline Santander & 47,02 & 33,20 \\
\hline Toledo & 19,78 & 36,30 \\
\hline Badajoz & 36,88 & 54,18 \\
\hline Logroño & 36,03 & 22,12 \\
\hline Bilbao & 100,09 & 14,30 \\
\hline Córdoba & 84,12 & 18,96 \\
\hline Alicante & 79,32 & 22,16 \\
\hline Vigo & 75,27 & 34,84 \\
\hline Gijón & 66,83 & 39,61 \\
\hline Hospitalet de Llobregat & 71,42 & 37,17 \\
\hline Santa Cruz de Tenerife & 51,63 & 54,10 \\
\hline
\end{tabular}

Fuente: Elaboración propia. 
La «Huella Hídrica» de la señalada eficiencia muestra notables diferencias espaciales entre las ciudades españolas recogidas en el estudio. Las hay que rondan el mínimo técnico, como Madrid y Barcelona que, según los datos de la tabla 7, registraron en el año 2015 unos datos de 7,55\% y el 8,16\%, respectivamente. En el otro extremo encontramos Badajoz, Toledo, Las Palmas o Santa Cruz de Tenerife, poniendo de manifiesto la necesidad de realizar importantes inversiones para evitar las pérdidas, y sus consecuencias.

La gestión sostenible e integrada de los recursos hídricos es uno de los temas globales más complicados para una empresa de distribución de agua potable. El agua dulce es un recurso limitado y valioso, que hay que preservar y usar en su justa medida. Dado que el agua es un recurso escaso debe ser gestionada de una manera eficiente, cuyo objetivo principal es que el máximo volumen de agua que entra en la red llegue a sus puntos de consumo, minimizando las pérdidas en la mayor medida posible ${ }^{6}$.

\section{Conclusiones}

Las ciudades generan una forma de ocupación más intensiva y diversificada que otros territorios, en cuanto a usos del suelo y actividades, y, por ende, son los principales centros de producción y generación de bienes y servicios, así como de consumo. Puesto que las materias primas necesarias para la producción de esos bienes y servicios consumidos y transferidos en las ciudades, no se producen en su integridad en el mismo territorio de la urbe, y en la mayoría de los casos, ni su entorno inmediato, en consecuencia, parece que son las ciudades las mayores generadoras de «Huella Hídrica» para sí mismas y para terceras regiones. $\mathrm{Y}$ es que los recursos hídricos — concebido como parte del capital natural - , son fundamentales para la supervivencia y para el desarrollo de cualquier región, por lo que ha de ser conservado mediante buenas prácticas urbanas

El «Agua Virtual» y la «Huella Hídrica» han de convertirse en indicadores fundamentales para optimizar las políticas de gestión y planificación de los recursos hídricos que permitan un uso eficiente y eficaz de los recursos hídricos en el ámbito urbano, en particular, y el desarrollo sostenible, en general.

${ }^{6}$ Durán, J.A. y Moral, J. (2015). «Gestión de las redes de abastecimiento. Control del Agua No Registrada (ANR)». IV Jornadas de Ingeniería del Agua La precipitación y los procesos erosivos Córdoba, 21 y 22 de Octubre 2015 
El consumo y comercio de «Agua Virtual». No debemos olvidar que en lo concerniente a los países que disponen de mayores niveles de comercio de recursos hídricos, a través del «Agua Virtual», son, en general, aquellos de mayores tasas de población urbana (si bien, este aspecto merecería un estudio aparte, que analizara con precisión la correlación nivel de urbanización, modelo territorial urbano y «Huella Hídrica»).

El consumo doméstico «per cápita» del agua parece no estar relacionado -al menos estadísticamente- con la mayoría de los indicadores básicos urbanos contemplados (la densidad de población, la extensión territorial, el tamaño medio de las viviendas, y las tasas de desempleo, no parecen incidir de manera directa sobre este consumo; en todos ellos, y en el conjunto de las ciudades que nos ofrece la fuente, hemos obtenido unos coeficientes de correlación muy débiles).

La «Huella Hídrica» de producción es tanto más elevada cuanto mayor es el puesto de la ciudad en la jerarquía urbana en nuestro modelo territorial, a nivel total, y con una relación no muy clara en cuanto al rango en el sistema urbano nacional, consecuencia cuasi directa del tamaño e importancia de la población, ya que si se relaciona jerarquía con «Huella Hídrica» per cápita, la relación no es tan ajustada.

\section{Bibliografía}

AEAS (2016). «XIV Estudio Nacional de Suministro de Agua Potable y Saneamiento en España, 2016».

Aquastat-FAO (2003). «Food and Agriculture Organization of the United Nations», Rome, Italy.

Banco Mundial. Borealis Group (2009). «Water Footprint». (2011). GNI per capita, Atlas method (current US\$).

Box \& Harrison (1992), Citado por Miracle, MR en «La Construcción de la ciudad sostenible». Consideraciones y casos en torno al ciclo del agua. http://habitat. aq.upm.es/cs/p3/a018.html.

Cabrera, E. (2008). «El suministro urbano de en España». Fundación Nueva Cultura del Agua. Panel Científico-Técnico de seguimiento de la Política de Aguas, Convenio Universidad de Sevilla-Ministerio de Medio Ambiente.

Carta de las Ciudades Europeas hacia la Sostenibilidad. Aalborg (1994).

Chapagain, A.K. y Orr, S. (2009). «UK Water Footprint: The impact of the UK's food and fibre consumption on global water resources», WWF-UK, Godalming, UK.

Chapagain, A.K. y Orr, S. (2009). «An improved water footprint methodology linking global consumption to local water resources», Journal of Environmental Management 90: 1219-1228. 
Chapagain, A.K. y Hoekstra, A.Y. (2004). «Water footprints of nations», Value of Water Research Report Series No. 16, UNESCO-IHE, Delft, the Netherlands.

De Miguel García, A. (2007) «Estimación del flujo de agua virtual entre las comunidades de CastillaLa Mancha y Murcia», MSc thesis, Univ. de Alcalá - Univ. Rey Juan Carlos.

Durán, J.A. y Moral, J. (2015). «Gestión de las redes de abastecimiento. Control del Agua No Registrada (ANR)». IV Jornadas de Ingeniería del Agua La precipitación y los procesos erosivos Córdoba, 21 y 22 de Octubre 2015

EUROSTAT. «Urban Indicator for Core Cities».

García Alvarado, J.M. (2000). «El modelo metropolitano madrileño: enjuiciamiento desde la perspectiva del V Programa de Acción de la C.E. en materia de Medioambiente. Apuntes metodológicos». Observatorio Medioambiental, No 3.

Hoekstra, A.Y. et alii. (2009). «Water footprint accounting, impact assessment, and lifecycle assessment». Proceedings of the National Academy of Sciences 106(40): E114.

Hoekstra, A.Y. et alii. (2011). «The water footprint assessment manual: setting the global standard», Earthscan, London.

Institutos Nacionales de Estadística (INE, 2016).

Jerez Mir, C. (2011). «¿Qué es el urbanismo?», Granada, Universidad de Granada., 166pp.

Lynch, K. (1965). «The City as Environment». in Cities, Scientific American. New York: Alfred A. Knopf, Inc., 1965, pp. 192-202.

Madrid, C. (2007) «Hidratar el Metabolismo Socioeconómico: Los Flujos de Agua Virtual y el Metabolismo Hídrico. Una aproximación al sector hortofrutícola Andaluz», MSc thesis, Universidad Autónoma de Barcelona.

Ministerio de Agricultura, Alimentación y Medio Ambiente. (2011). «Programa A.G.U.A.»

Disponible en: http://www.mma.es/secciones/agua/entrada.htm

Ministerio de Agricultura, Alimentación y Medio Ambiente. (2011). «Agua: Seguridad de Presas y Embalses». Disponible en: http://www.marm.es/es/agua/temas/seguridad-de-presas-y-embalses/

Rodríguez Casado, R.; Garrido, A.; Llamas, M.R. and Varela-Ortega, C. (2008) «La huella hidrológica de la agricultura española». Papeles de Agua Virtual n. ${ }^{\circ} 2$, Fundación Marcelino Botín, Santander, ISBN 978-84-96655-25-6, 38 pp.

Rodríguez Casado, R; Novo, P y Garrido A (2009). «La huella hídrica de la ganadería española». Papales de Agua Virtual, número 4. Fundación Marcelino Botín.

Rueda Palenzuela Salvador (1999). «Modelos e Indicadores Para Ciudades Más Sostenible. Taller sobre Indicadores de Huella y Calidad Ambiental Urbana». Departament de Medi Ambient de la Generalitat de Catalunya.

Sotelo Navalpotro, J.A. (2009). «Las lógicas ilógicas del agua». Tribuna Complutense.

Sotelo Navalpotro, J. A., Olcina Cantos, J., Tolón Becerra, A., Lastras Bravo, X., García Quiroga, F, Sotelo Pérez, Mª . (2010). «Huella Hídrica española en el contexto del cambio ambiental global», pp. 218. Fundación Mapfre. 
Sotelo Navalpotro, J. A., Olcina Cantos, J., Tolón Becerra, A., García Alvarado, J. Mª ., Lastras Bravo, X., García Quiroga, F, Sotelo Pérez, Mª., Sotelo Pérez, I. (2011). «Huella hídrica, desarrollo y sostenibilidad en España», pp. 425. Fundación Mapfre Sotelo Navalpotro, J.A., Tolón Becerra, A. y Lastras Bravo, X. (2011). «Indicadores por y para el desarrollo sostenible, un estudio de caso». Estudios Geográficos. Volumen 72, no 271. pp. 611-65

Sotelo Navalpotro, J. A., Sotelo Pérez, M. y García Quiroga, F. (2011). «Análisis «costebeneficio» $y$ «coste-eficiencia» de la Huella Hídrica en España». Observatorio Medioambiental. Pp. 225-254.

Sotelo Navalpotro, J. A., Olcina Cantos, J., García Quiroga, F. y Sotelo Pérez, M. (2012, B). «Huella Hídrica de España y su diversidad territorial». Estudios Geográficos, volumen 73, pp. 239-272.

Sotelo Navalpotro, J. A. (2016). «Tras las "Huellas" del agua en España». Boletín de la Real Sociedad Geográfica, tomo CLI, pp. 259-288.

Sotelo Pérez, M. (2012). "Economía y Huella Hídrica en España (I)". Apuntes de Medio Ambiente, Boletín del Ilustre Colegio de Doctores y Licenciados, número 12.

Sotelo Pérez, M. (2013). "Economía y Huella Hídrica en España (II)" Apuntes de Medio Ambiente, Boletín del Ilustre Colegio de Doctores y Licenciados, número 13.

Sotelo Pérez, M. (2015). "Cuestiones de escala en el ámbito de la Huella Hídrica". Observatorio Medioambiental, vol. 18, pp. 9-37. ISSN: 1139-1987

Vázquez Barquero, A. (2005). "Las nuevas fuerzas del desarrollo". Antoni Bosch Editor Barcelona. ISBN: 84-95348-16-0

Water Footprint Network (2010). "Water Footprint - Glossary".

William E. Rees (1992). "Ecological footprints and appropriated carrying capacity: what urban economics leaves out". Environment and Urbanization October 1992 vol. 4 no. 2 121-130.

Fecha de recepción: 15 de diciembre de 2016.

Fecha de aceptación: 26 de febrero de 2018. 